\title{
Hydrogen Storage in a Prototypical Zeolitic Imidazolate Framework-8
}

\author{
Hui Wu, ${ }^{\dagger, \ddagger}$ Wei Zhou, ${ }^{\dagger, \S}$ and Taner Yildirim*, ${ }^{\star}, \S$ \\ NIST Center for Neutron Research, Gaithersburg, Maryland 20899-8562, Department of Materials Science and \\ Engineering, University of Maryland, College Park, Maryland 20742, and Department of Materials Science and \\ Engineering, University of Pennsylvania, Philadelphia, Pennsylvania 19104
}

Received December 21, 2006; E-mail: taner@nist.gov

Zeolitic imidazolate frameworks (ZIFs) are a new class of nanoporous compounds which consist of tetrahedral clusters of $\mathrm{MN}_{4}$ ( $\mathrm{M}=\mathrm{Co}, \mathrm{Cu}, \mathrm{Zn}$, etc.) linked by simple imidazolate ligands. ${ }^{1,2} \mathrm{As}$ a subfamily of metal-organic frameworks (MOFs), ZIFs exhibit the tunable pore size and chemical functionality of classical MOFs. At the same time, they possess the exceptional chemical stability and rich structural diversity of zeolites. ${ }^{2}$ Because of these combined features, ZIFs show great promise for hydrogen storage applications. However, in contrast to a large number of extensive studies for other MOFs, ${ }^{3-5}$ no experimental data concerning the nature of $\mathrm{H}_{2}$ ZIF interactions and the manner in which hydrogen molecules are adsorbed have been reported yet. Such fundamental studies hold the key to optimizing this new class of ZIF materials for practical hydrogen storage applications. In particular, the major adsorption sites and their binding energies are the key features of a system that determine its adsorption properties at a given temperature and pressure.

ZIF8 is a prototypical ZIF compound $\left(\mathrm{Zn}(\mathrm{MeIM})_{2}\right.$, MeIM = 2-methylimidazolate) with a SOD (sodalite) zeolite-type structure, exhibiting an interesting nanopore topology formed by four-ring and six-ring $\mathrm{ZnN}_{4}$ clusters as shown in Figure 1. Since the nanopores are only accessible through narrow six-ring funnel-like channels (Figure 1a), one wonders how $\mathrm{H}_{2}$ molecules are adsorbed, where the major binding sites are, and what the binding energies are. Herein, using the difference Fourier analysis of neutron powder diffraction data along with first-principles calculations, we provide answers to these questions for the first time. Surprisingly, the strongest adsorption sites that we identified (see Figure 1b) are directly associated with the organic linkers, instead of the triangular faces of the $\mathrm{ZnN}_{4}$ tetrahedra (i.e., metal sites), in strong contrast to other MOFs, where the faces of the metal-oxide tetrahedra are typically the primary adsorption sites. At high $\mathrm{H}_{2}$ loading, the ZIF8 structure is capable of holding up to $28 \mathrm{H}_{2}$ molecules (i.e., $4.2 \mathrm{wt}$ $\%)$ in the form of highly symmetric novel three-dimensional (3D) interlinked $\mathrm{H}_{2}$-nanoclusters.

ZIF8 was synthesized using a solvothermal method as described in ref 2. Neutron powder diffraction data were collected on the high-resolution neutron powder diffractometer (BT-1) at NIST Center for Neutron Research. Because of the large incoherent cross section of $\mathrm{H}_{2}$, adsorption was studied as a function of $\mathrm{D}_{2}$ concentration per ZIF8 molecular formula $\left(\mathrm{Zn}_{6} \mathrm{~N}_{24} \mathrm{C}_{48} \mathrm{H}_{60}\right)$. Target amounts of $\mathrm{D}_{2}$, that is, 3,16 , and $28 \mathrm{D}_{2}$ per $6 \mathrm{Zn}$, were loaded into the ZIF8 sample at $70 \mathrm{~K}$. One $\mathrm{H}_{2} / 6 \mathrm{Zn}$ corresponds to $\approx 0.15$ wt $\%$ hydrogen uptake. The sample was then cooled to $30 \mathrm{~K}$ at which point the $\mathrm{D}_{2}$ was completely adsorbed. Once the system was equilibrated at $30 \mathrm{~K}$, the sample was further cooled to $3.5 \mathrm{~K}$ before the diffraction measurement. No evidence of solid deuterium was

\footnotetext{
$\dagger$ NIST Center for Neutron Research.

\# University of Maryland.

$\S$ University of Pennsylvania.
}
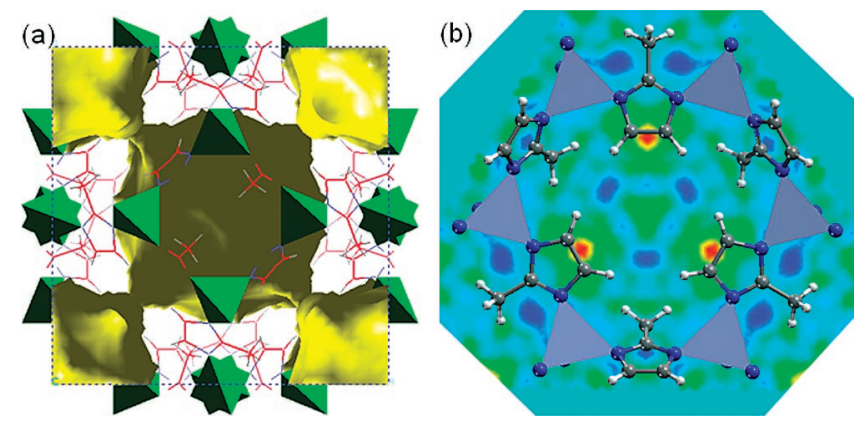

Figure 1. (a) (001) view of refined crystal structure of ZIF8 host lattice from neutron powder diffraction along with the available free space (pore structure) for $\mathrm{H}_{2}$ occupation, based on van der Waals interactions. (b) A (111) view of the real-space Fourier-difference scattering-length density superimposed with six-ring pore aperture of the ZIF8 structure, indicating the location of the first adsorption sites (red-yellow regions).

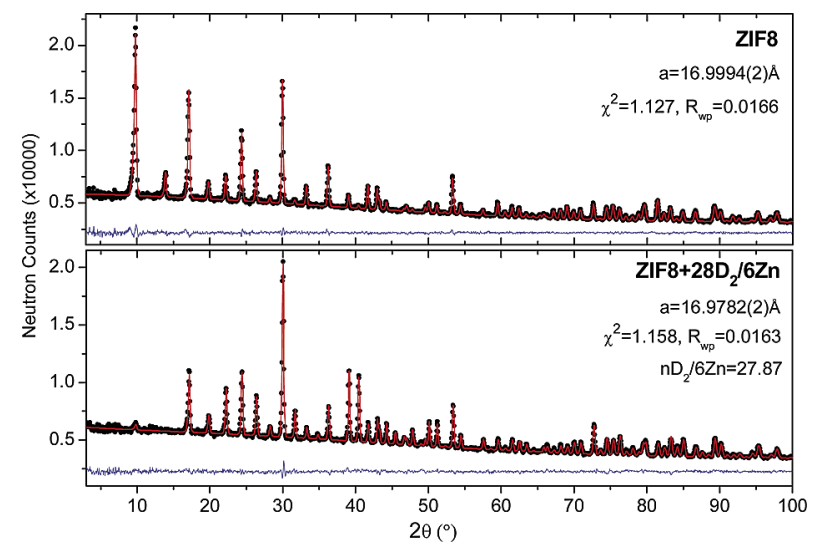

Figure 2. Observed (dots), refined (line), and difference (noisy line) neutron powder diffraction profiles $(\lambda=2.079 \AA$, $3.5 \mathrm{~K})$ for ZIF8 host lattice (space group $I 4 \overline{3} \mathrm{~m}$ ) and ZIF8 with $\mathrm{D}_{2}$ loading of $28 \mathrm{D}_{2} / 6 \mathrm{Zn}$.

observed on the structural refinement of the $\mathrm{D}_{2}$ loaded samples, indicating all $\mathrm{D}_{2}$ was adsorbed into the ZIF8.

The top panel of Figure 2 shows the neutron diffraction data from the ZIF8 bare material measured at $3.5 \mathrm{~K}$. The refined lattice parameters and atomic positions of $\mathrm{Zn}, \mathrm{N}$, and $\mathrm{C}$ agree well with previously reported room-temperature X-ray diffraction results. ${ }^{2}$ The high-resolution neutron diffraction data also enabled us to unambiguously determine the orientation (i.e., the hydrogen positions) of the methyl group, which was not possible in the X-ray measurement. We note that the methyl group orientation and associated tunnel-splitting is a very sensitive probe for determination of the guest-host interactions. ${ }^{6}$

For comparison, the neutron diffraction patterns from ZIF8 with the following $\mathrm{D}_{2}$ concentrations: $n \mathrm{D}_{2}=3,16,28$ per $6 \mathrm{Zn}$ are also shown in Figure 2 (also see Supporting Information). Using the model of the refined ZIF8 host structure, we performed Rietveld 


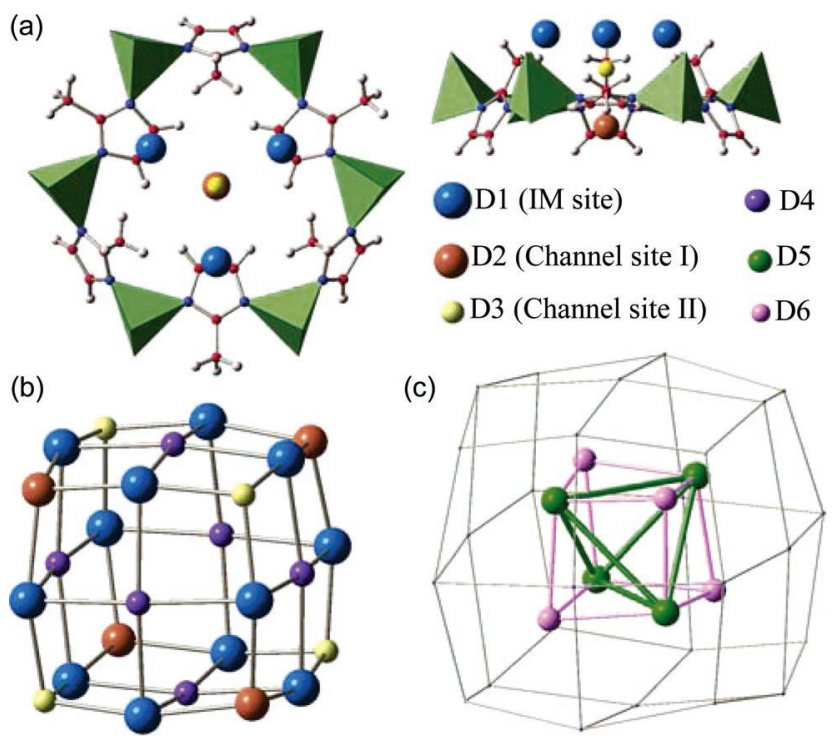

Figure 3. The hydrogen adsorption sites obtained from Fourier difference analysis: (a) top and side views of first three adsorption sites near a $\mathrm{Zn}$ hexagon opening; (b) pseudocubic nanocage formed by D1, D2, and D3 sites; (c) tetrahedron-like nanocage formed by D5 and D6 sites.

structure refinements on the $\mathrm{D}_{2}$ adsorbed samples. The Fourier difference method was used to find the scattering-length density distribution for $\mathrm{D}$ atoms based on the refinements ignoring the adsorbed $\mathrm{D}_{2}$ molecules. Figure $1 \mathrm{~b}$ is an example of such Fourier difference plot, which clearly shows that the initial adsorption site (i.e., D1) is on top of the MeIM organic linker and close to the $\mathrm{C}=\mathrm{C}$ bond, thus termed "IM site".

Upon $\mathrm{D}_{2}$ loading, the order of site filling in ZIF8 was determined to be sequential from D1 to D6, shown in Figure 3. The already mentioned D1 site and the second site (D2) are both determined from the initial loading of $3 \mathrm{D}_{2} / 6 \mathrm{Zn}$. The latter is located at the center of the channel of the six-ring opening (termed "channel site I"). The distances between the $\mathrm{H}_{2}$ center of mass and the corresponding $\mathrm{C}=\mathrm{C}$ bond are about 3 and $3.4 \AA$ for D1 and D2 sites, respectively. For this particular $\mathrm{D}_{2}$ concentration, IM site occupancy is $\sim 21 \%$ while it is $\sim 10 \%$ for the channel site, implying slightly higher binding energy for D1 than for D2 sites.

For $16 \mathrm{D}_{2} / 6 \mathrm{Zn}$ loading, the D1 and D2 sites are nearly fully occupied, and the third adsorption site (D3) starts to populate. The third site is also at the center of the six-ring opening but located on the other side of the complex, so we termed it "D3: channel site II". This site is less favorable than D2 channel site I owing to the presence of the methyl group, mainly a geometry effect. Interestingly, the first three adsorption sites discussed above form a pseudocubic "nanocage" with the edges slightly bent toward the ZIF8 framework as shown in Figure 3c. We also observed a small amount $(\sim 5 \%)$ of $\mathrm{D}_{2}$ adsorbed at a fourth site (D4) located at the face center of the $\mathrm{H}_{2}$-nanocage.

With further $\mathrm{D}_{2}$ loading, the first three adsorption sites were almost completely occupied. For the maximal loading of $28 \mathrm{D}_{2} /$ $\mathrm{Zn}$, the fourth site discussed above as well as two additional sites (D5 and D6) close to the center of the void in ZIF8 (inside the nanocage formed by the first three sites) are progressively occupied. The nearest neighbor distances between these adsorption sites are about $3.04 \AA$ (see Supporting Information), significantly shorter than the $3.6 \AA$ found in solid $\mathrm{H}_{2}$. Self-assembled 3D interlinked hydrogen nanostructures with short intermolecular distances were previously observed ${ }^{3}$ in MOF5 and seem to be a common novel feature of these nanoporous metal-hybrid systems. Note that our maximum loading of $4.2 \mathrm{wt} \%$ (absolute adsorption capacity) obtained at $30 \mathrm{~K}$ is higher than the reported $3.1 \mathrm{wt} \%$ (excess adsorption capacity) obtained at $77 \mathrm{~K}$ and 80 bar, ${ }^{2}$ as expected for adsorption at lower temperature.

To understand the hydrogen host-lattice interactions further, we also performed total-energy calculations from density functional theory (DFT) using the plane-wave implementation of the localdensity approximation to $\mathrm{DFT}^{7}$ (see Supporting Information). Consistent with experimental observation, our calculations suggested that the IM and channel site I are the most energetically stable adsorption centers. The calculated $\mathrm{H}_{2}$ binding energies for these two sites are 170 and $147 \mathrm{meV}$, respectively, in agreement with the larger population of the IM site at low $\mathrm{D}_{2}$ loading. We also found about $20 \mathrm{meV}$ variation in the binding energy with $\mathrm{H}_{2}$ orientation, suggesting that interesting hindered-quantum rotational dynamics could be present in the $\mathrm{H}_{2}$-ZIF8 system. Note that the binding energies derived from DFT may be overestimated. However, the relative magnitude should be valid, and indeed a good agreement between calculation and experiment was found in both this work and previous work ${ }^{3}$ on other types of MOFs, in terms of correctly predicting the relative strength of various adsorptions sites.

In conclusion, we obtained detailed structural information such as orientation of the methyl groups, $\mathrm{H}_{2}$ adsorption sites, and binding energies in the ZIF8 structure for the first time. The imidazolate organic linker is primarily responsible for adsorption in contrast to metal oxide-based MOFs. This suggests that modification of the linkers rather than metal types in ZIFs is more important to optimize these materials for higher storage capacity. At high concentration of hydrogen loading, ZIF8 is able to hold hydrogen molecules up to $4.2 \mathrm{wt} \%$ as self-assembled nanostructures with relatively short intermolecular distances compared to solid hydrogen. This suggests that ZIFs can be also used as an ideal template host-material to generate molecular nanostructures with interesting properties.

Acknowledgment. This work was partially supported by DOE BES Grant No. DE-FG02-98ER45701 (W.Z., T.Y.) and DOE EERE Grant No. DE-AI-01-05EE11104 (H.W.).

Supporting Information Available: Detailed information about the Rietveld refinement and the crystallographic data for ZIF8- $n \mathrm{D}_{2}(n$ $=0,3,16$, and 28), and detailed information about the DFT calculations. This material is available free of charge via the Internet at http://pubs.acs.org.

\section{References}

(1) Huang, X. C.; Lin, Y. Y.; Zhang, J. -P.; Chen, X. M. Angew. Chem., Int Ed. 2006, 45, 1557-1559.

(2) Park, K. S.; Ni, Z.; Cote, A. P.; Choi, J. Y.; Huang, R.; Uribe-Romo, F. J.; Chae, H. K.; O'Keefe, M.; Yaghi, O. M. Proc. Natl. Acad. Sci. U.S.A. 2006, 103, 10186-10191

(3) Yildirim, T.; Hartman, M. R. Phys. Rev. Lett. 2005, 95, 215504-215507.

(4) Peterson, V. K.; Liu, Y.; Brown, C. M.; Kepert, C. J. J. Am. Chem. Soc 2006, 128, 15578-15579

(5) Dinca, M.; Dailly, A.; Liu, Y.; Brown, C. M.; Neumann, D. A.; Long, J. R. J. Am. Chem. Soc. 2006, 128, 16876-16883.

(6) Press, W. Single-Particle Rotations in Molecular Crystals; SpringerVerlag: Berlin, 1981; Vol. 92.

(7) Baroni, S.; Dal Corso, A.; de Gironcoli, S.; Giannozzi, P. Plane-Wave Self-Consistent Field. http://www.pwscf.org.

JA0691932 